
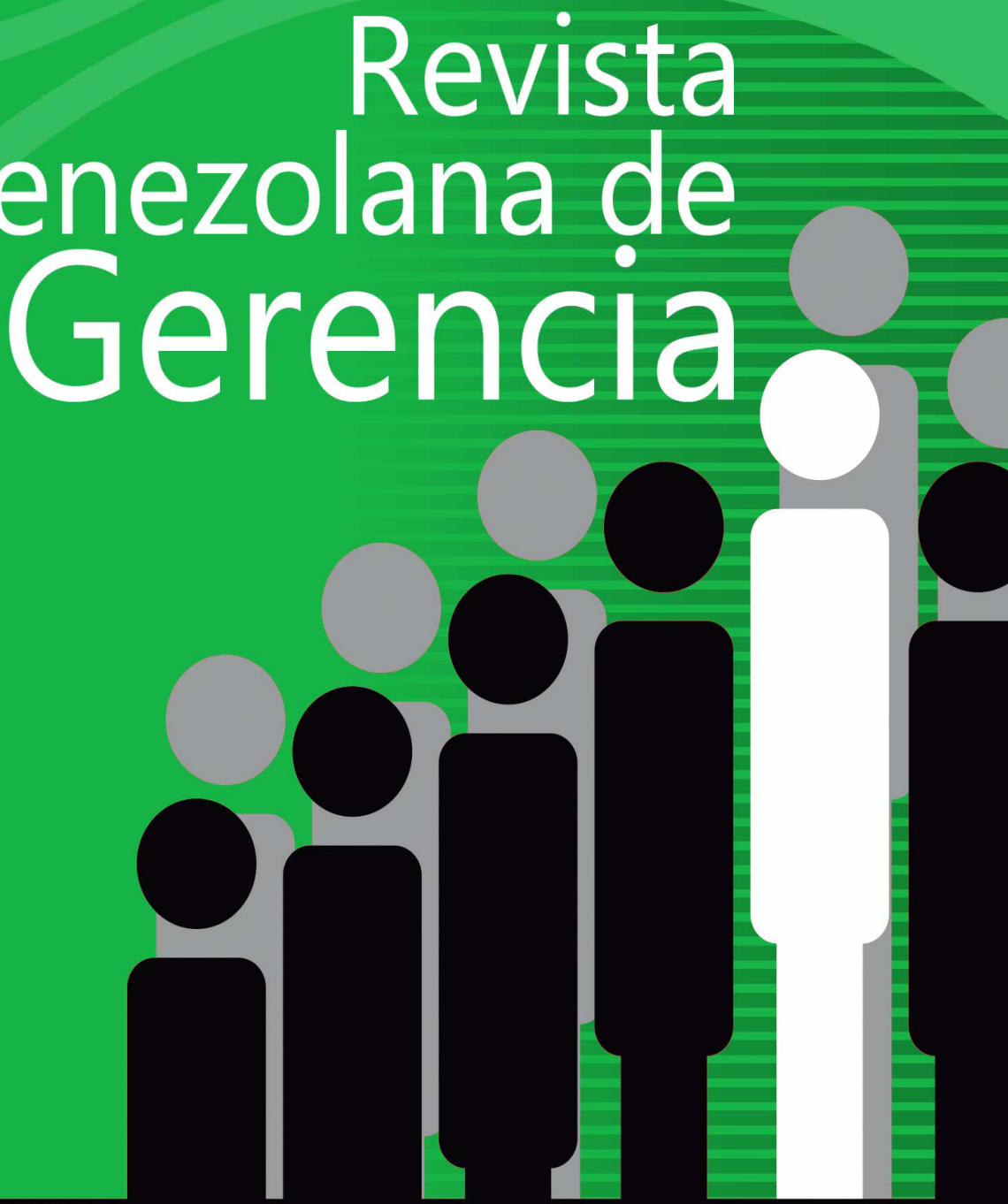


\title{
Gobernanza, enfermería, seguridad del paciente en una empresa social del estado colombiano
}

\author{
Mera García, Manuela ${ }^{1}$ \\ Thorpt Hurtado, Carmen del Pilar ${ }^{2}$ \\ González Osorio, María Fernanda ${ }^{3}$
}

\section{Resumen}

La investigación analizó el papel de la gobernanza en el Programa de Seguridad del Paciente de una Empresa social del Estado, del suroccidente colombiano, desde el equipo de enfermería que lidera el programa. Para ello, el estudio tuvo un enfoque cualitativo, de tipo exploratorio interpretativo, mediante entrevistas semiestructuradas a enfermeras líderes, apoyado con fuentes secundarias. Las entrevistas contaron con una guía previamente validada por expertos y fueron grabadas y organizadas en categorías temáticas. El análisis destaca las características del equipo líder de enfermería, su influencia en el programa, la toma de decisiones y los principios de gobernanza que centran su actuar, las normas regulares e informales contempladas en el Programa, así como los espacios, interacciones y procesos del equipo con relación a la atención segura de los pacientes. Los resultados de la investigación identificaron cada uno de los integrantes del equipo, de acuerdo a su formación y nivel de participación en el programa. A pesar de las debilidades en recursos, el grupo mostro una visión común de acuerdo a los principios que impactan la cultura de seguridad del paciente. Es un equipo con mucha experiencia y formación posgradual, pero con poca formación en gestión y políticas públicas, ejes

\footnotetext{
Recibido: 11-09-19 Aceptado: 10-11-19
}

Enfermera, docente Universidad Santiago de Cali, Magister en Gestión Pública. ORCID 0000-0002-15638803. Correo: manuela.mera00@usc.edu.co

2 Enfermera. Magister en Gestión Pública, Universidad Santiago de Cali. ORCID: 0000-0003-1961-0442. Correo: carmen.thorpt00@usc.edu.co

3 Docente Universidad Santiago de Cali, Magister en Educación y Desarrollo Humano, Universidad San Buenaventura. Magister en Filosofía Universidad del Valle. ORCID: 0000-0001-5741-8561.Correo: mafegonzalez@usc.edu.co 
importantes en el actuar la atención segura en el programa, eje central de la Política de Seguridad del paciente. También que el equipo líder de enfermería debe actuar en red y, por tanto, debe mejorarse la transversalidad de la cultura de seguridad del paciente en la institución, fortaleciendo la cohesión con el equipo médico, el de trabajo social, y otros equipos que también garantizan la atención segura al usuario. Una mayor presencia del comité interinstitucional de eventos adversos, debe considerarse como elementos claves de la cultura organizacional.

Palabras clave: Gobernanza; enfermería; programa de seguridad del paciente; empresa social

\title{
Governance, nursing, patient safety in a social enterprise in the Colombian state
}

\begin{abstract}
The research analyzed the role of governance in the Patient Safety Program of a social enterprise of the State, of the Colombian southwest, from the nursing team leading the program. For this, the study had a qualitative approach, of exploratory interpretative type, through semi-structured interviews with leading nurses, supported by secondary sources. The interviews had a guide previously validated by experts and were recorded and organized in thematic categories. The analysis highlights the characteristics of the leading nursing team, its influence on the program, decision-making and governance principles that focus its actions, the regular and informal standards contemplated in the Program, as well as the spaces, interactions and processes of the program. team regarding the safe care of patients. The research results identified each of the team members, according to their training and level of participation in the program. Despite the weaknesses in resources, the group showed a common vision according to the principles that impact the patient's safety culture. It is a team with a lot of experience and postgraduate training, but with little training in management and public policies, important axes in acting safe attention in the program, central axis of the Patient Safety Policy. Also that the leading nursing team must act in a network and, therefore, the transversality of the culture of patient safety in the institution should be improved, strengthening cohesion with the medical team, the social work team, and other teams that also guarantee Safe attention to the user. A greater presence of the inter-institutional committee of adverse events should be considered as key elements of the organizational culture.
\end{abstract}

Keywords: Governance; nursing; patient safety program; social enterprise 


\section{Introducción}

El concepto de gobernanza está estrechamente relacionado con cambios en los diferentes campos de las ciencias sociales, en el campo de la salud se la concibe como un modelo que permite el desarrollo de iniciativas participativas entre el Estado y la sociedad, tanto en su esfera pública como privada, que interactúan con diversos roles entre sectores involucrados para resolver problemas en la prestación del servicio de salud.

La gobernanza resulta ser una alternativa a la solución de problemas de la sociedad a partir de la redistribución del poder estatal y la capacidad de autonomía de las instituciones para su desarrollo, a la vez que contribuye a disminuir la brecha que existe entre lo que se anuncia como política y lo que se ejecuta, dando respuesta a la problemática local con modelos más incluyentes, mejorando la utilización y capacidad de recaudo de los recursos y por consiguiente de impacto en el bienestar.

De otra parte, la atención segura y humana en salud es un elemento trasversal a todas las instituciones de salud sin discriminación de su carácter público o privado, nivel local, regional o nacional; los Programas de Seguridad del Paciente, se articulan a todos los procesos institucionales de manera integral para detectar situaciones tempranas de riesgo e inmediatamente intervenir como equipo de salud.

Los Ministerios de Salud de Argentina, Colombia, Costa Rica, México y Perú con el apoyo y asesoría de la OMS, OPS y del gobierno español, realizaron el estudio IBEAS para medir la prevalencia de eventos adversos en las instituciones de salud participantes; dicho estudio concluye, que es necesario para implementar la política de seguridad del paciente en las instituciones de salud, unificar equipos en diferentes niveles de atención. (OMS,2009). De otro lado, Gómez et al, (2011) en el estudio "Cultura de seguridad del paciente por personal de enfermería en Bogotá, Colombia", concluye la importancia del equipo de enfermería en la identificación de aspectos relevantes del proceso del cuidado que favorezcan la disminución de riesgos y mejoren la cultura de seguridad del paciente, a partir de: la coordinación en los diferentes niveles de autoridad; la comunicación eficaz dentro de las unidades; la toma de decisiones y la autonomía de estas unidades para una atención segura; de igual manera, consideró que el personal insuficiente y el alto flujo de personal, ocasionan riesgos en la atención segura.

En Colombia, la Política de Seguridad del Paciente se expide en junio del 2008, por el Ministerio de Salud y la Protección Social, como eje trasversal de los componentes del Sistema Obligatorio de Garantía de Calidad. En la empresa Social del estado, esta política, se recoge en el Programa de Seguridad del Paciente, creado en el 2012 y actualizado en 2015 con la inclusión de las 10 metas de seguridad del paciente con miras a la acreditación de los servicios de salud. Desde su inicio, el Programa es liderado por el área de enfermería, como programa, contiene los procesos que propenden por minimizar el riesgo de sufrir un evento adverso en el proceso de atención en salud o de mitigar los efectos de dicha atención. Cuenta con los principios orientadores que están en concordancia con los definidos en los lineamientos para la implementación de la política de seguridad del paciente del 
Ministerio de Salud y de la Protección Social: atención centrada en el usuario, eficacia, validez, cultura de seguridad del paciente, y 3 líneas de acción, dos de las cuales son de niel gerencial y una donde participa el equipo de enfermería como es Conformación de Comité técnico por la Seguridad del paciente (Minsalud, 2008, 2011, 2012).

Sin embargo, la crisis estatal del sistema de salud y factores internos de la Empresa Social del Estado, evidenciaron una crisis financiera y estructural en la institución, y la obligaron a un plan de austeridad (Ley 550, 1999), optimización de recursos, racionalización de gastos de funcionamiento e inversión. Estos cambios, por tanto, afectaron en cantidad, cualificación y reasignación de funciones, al personal de enfermería. (Draga et al, 2016)

Es posible entonces, que los cambios sufridos por el personal de enfermería de la institución, producto de la crisis y la reestructuración, repercutieran en su actuar como equipo líder de un programa tan importante. Por ello, el análisis de la gobernanza desde la perspectiva del equipo de enfermería, se consideró pertinente.

Para ello, La investigación caracterizó al equipo de enfermería que lidera el programa se seguridad del paciente, examinó su percepción frente a la aplicación de los principios de gobernanza e identificó los espacios de interacción, normas y procesos seguidos por el grupo, como actores de primera línea en la atención segura en la Empresa Social, ESE.

Se diseñó un estudio cualitativo de tipo exploratorio e interpretativo, a partir de entrevistas semiestructuradas a un grupo de enfermería comprometido en el liderazgo del Programa de Seguridad del paciente, en una Empresa Social del Estado. El grupo estuvo compuesto por:1) Enfermeras coordinadoras de área (EECA) quienes direccionan las estrategias de seguridad del paciente en los servicios de hospitalización asignados. 2) Enfermeras especialistas con funciones precisas frente a la meta de seguridad del paciente (EE); y 3) Enfermeras profesionales (EP) que responden a las funciones de asistencia directa y del programa de seguridad en salas de hospitalización. Se realizaron 9 entrevistas.

Como instrumento para esta investigación se elaboró una guía de entrevista, las preguntas se revisaron y aprobaron por tres expertos mediante una rejilla de evaluación que valoró la claridad y pertinencia de las preguntas. Las entrevistas fueron realizadas en los puestos de trabajo de los actores de la Empresa Social por las dos investigadoras principales del estudio, fueron grabadas, previo consentimiento informado, para un total de 9 entrevistas. Para el análisis se diseñaron categorías deductivas teniendo en cuenta los principios de gobernanza de enfermería y las categorías propuestas por Hufty (2010) para el Marco Analítico de Gobernanza en salud: Caracterización de actores; y espacios de interacción, normas y procesos en el programa de seguridad del Paciente.

\section{Sobre la gobernanza y la gobernanza en salud}

La gobernanza es un término de múltiples definiciones como fenómeno social, es descrita por autores en muchas disciplinas que de una y otra manera conducen a la solución de problemas sociales con aplicación en estudios e investigaciones por todas las ciencias, con el propósito de generar 
trasformación e impactos en la sociedad moderna. Su uso crece en diversos ámbitos, globales y locales, que dan a la gobernanza un dinamismo que se asocian al diseño e implementación de las políticas públicas.

El enfoque de gobernanza surge a partir de los años 80 como una forma de entender la relación del estado y a sociedad, ante las bajas respuestas del estado frente a la descentralización, las demandas sociales y la globalización. (Prats,2005; Aguilar,2003,2007; Kooiman,2005).

A nivel de los organismos Internacionales, prima una visión de gobernanza desde la perspectiva de desarrollo económico, social y político, y como un método para evaluar resultados tendientes a la estabilidad política, ausencia de violencia, efectividad gubernamental, estado de derecho, control de la corrupción y calidad con base a principios de la gobernanza, (Banco Mundial,2017). (La Organización para la Cooperación y el Desarrollo, OECD, 2017)

Pero más que un enfoque centrado en el estado, su gobierno y su relación jerárquica con la sociedad; dentro de la Nueva Gestión Pública, interesa el enfoque de la nueva gobernanza, también llamado Gobernanza moderna, el cual revisa de manera crítica la verticalidad de las interacciones entre el estado y la sociedad. Se ve la gobernanza un concepto que plantea que las políticas públicas dependen no solo del estado sino de actores con intereses distintos, que realizan funciones diferentes y en múltiples niveles (Subirats, 2010).

En este sentido Kooiman, (2005) colocando el énfasis en los actores, propone que la gobernanza replantea el papel del estado y de la sociedad como actor político, reconoce en la dinámica de lo público nuevos actores y la formación de redes en las que la acción colectiva se concibe de manera horizontal. Define la gobernanza como un instrumento para resolver los problemas sociales y políticos a partir de diferentes actores sociales, políticos, institucionales gubernamentales y no gubernamentales que interactúan entre sí de acuerdo a sus roles. (Kooiman, 2005)

Dentro de esta perspectiva centrada en los actores, se destacan las propuestas de Prats (2005), Aguilar $(2003,2010)$, y Hufty (2006), que resaltan el vínculo con lo social y no solamente con las capacidades del estado y la resolución de asuntos comunes a partir de la pluralidad e interacción de los actores.

Para Prats (2005), la gobernanza es flexible, multifacética y plural dependiendo de la actuación de los actores que a su vez cuentan con normas, principios y valores contemplados en los Estados democráticos. Para Aguilar, la gobernanza es un proceso en el cual los actores de una sociedad deciden sus objetivos de convivencia y la forma de coordinarse para realizarlos y direccionaros, Aguilar (2003) y para Hufty, la gobernanza es asumida como una herramienta analítica para la comprensión de la acción colectiva que organiza la interacción de los atores, la dinámica de los procesos y la reglas de juego, ya sean estas formales o informales, con las cuales una sociedad toma e implementa sus decisiones y determina sus conductas. Esto es aplicable tanto a nivel internacional $y$ global, como a nivel nacional y local. (Hufty, 2006)

La Organización Mundial de la Salud (OMS) la definió como "La participación de actores a los cuales les concierne la definición e implementación 
de políticas, programas y prácticas que promueven sistemas de salud equitativos y sostenibles" (WHO, 1998). Desde la gobernanza en salud, la investigación se identifica con la definió de gobernanza de Hufty (2010) como: “... los procesos de interacción entre los actores involucrados en un asunto público que llevan a la toma de decisiones o formulación de normas sociales presentes en cualquier sociedad" de Hufty (2010) y destaca para el caso de empresas o instituciones, un enfoque analítico que proporciona un marco para investigar y analizar los cambios de gobernanza en un contexto local, identificar los espacios y normas para la toma de decisiones y las interacciones para influir en estos espacios, así como los puntos críticos. Hufty (2010)

Consta de cuatro categorías analíticas: 1) los actores, formales e informales, reconocidos o no por la ley o las autoridades; 2) sus normas, son las reglas de juego de los procesos de gobernanza, pueden ser constitutivas, regulativas o criterios o valores que definen para este caso la política mundial de seguridad del paciente; 3) sus puntos nodales, que corresponden a los espacios físicos o virtuales de interface en donde convergen los problemas, procesos, actores y normas; se toman decisiones se concluyen acuerdos y se elaboran normas sociales, $y$; 4) los procesos, que consisten en sucesiones dinámicas de estados por los cuales pasan las interacciones entre actores, normas y puntos nodales. Los procesos, los actores y las normas pueden ser formales o informales que permiten evaluar la dirección por la cual evolucionan los procesos y de localizar los factores al cambio (Hufty, 2010).

Resultan importantes para mejorar la incidencia en las políticas y prácticas del sistema de salud, lograr una mejor comprensión de los procesos de gobernanza vinculados al desarrollo e implementación de políticas en salud; los estudios los estudios de Cardona et al, (2010), Díaz-Castro, et al,(2017), son investigaciones aplicadas del marco analítico de Hufty, describen cómo interactúan los sujetos sociales, qué normas acogen para interactuar y cuáles son los nodos de actuación más importantes para la propuesta de intervención que garanticen la continuidad de la política pública. Sobre la gobernanza en la práctica del enfermero a nivel hospitalario, Guedes dos Santos y Erdmann (2015), muestran por ejemplo, cómo la gobernanza en la práctica profesional del enfermero se basa en la gestión del cuidado y cómo se esfuerzan en resolver las limitaciones del soporte organizacional.

\section{Principios de la gobernanza}

Los principios de la gobernanza proporcionan una guía para la toma de decisiones en contextos cambiantes, plurales y de convergencia de diferentes intereses, (Agustí Cerillo i Martínez, 2005), mantienen buenas prácticas para una buena Gobernanza responsable y con calidad para todos (CIE, 2014), y permite una mirada a la atención segura más allá de la norma, con compromiso y cooperación de actores para sensibilizar, promover y coordinar acciones con logros efectivos, (Minsalud, 2008).

La presente investigación organiza los principios de gobernanza de acuerdo a la similitud, relacionando principios definidos por tres fuentes: los principios o lineamientos de la gobernanza enunciados por el Comité Internacional de Enfermería en el Plan Estratégico 2014 - 2018 (CIE, 2014) y los principios 
de la política de seguridad del paciente del Ministerio de Salud y de la Protección Social (Minsalud, 2008), de la siguiente manera:

\subsection{Participación, colaboración y enfoque centrado en el usuario}

Involucra a todos aquellos actores que ostenten intereses, recursos o visiones comunes que incorporan en las redes de actores, con las normas y pautas del programa; la colaboración entre actores con intereses, recursos o visiones comunes y el enfoque de atención centrado en el usuario es el eje alrededor del cual giran todas las acciones de seguridad del paciente Minsalud (2008).

\subsection{Transparencia, rendición de cuentas y validez.}

La transparencia se erige en un mecanismo a través del cual se manifiesta el principio de rendición de cuentas (accountability) de los poderes públicos; la transparencia tiene importantes consecuencias, puesto que permite incrementar la legitimidad democrática de las propias administraciones (Agustí Cerillo i Martínez, 2005). Los organismos de reglamentación deben estar abiertos y ser transparentes en sus procesos y deben comunicarse empleando un lenguaje claro, apoyar la participación de personas ajenas y poner a disposición del público la cantidad máxima de información, los procesos reglamentarios deben ofrecen un trato justo y equitativo a todas las partes involucradas, CIE (2014), Minsalud (2008), incluye el principio de validez como la necesidad de implementar metodologías y herramientas prácticas que garanticen la evidencia científica a la vez que integra la política de atención segura con el Sistema Obligatorio de Garantía de Calidad de la Atención en Salud de manera trasversal a todos sus componentes.

\subsection{Eficacia, eficiencia y seguridad del paciente.}

La eficacia hace años que ocupa un papel relevante en la agenda pública. La eficacia es la consecución del resultado que corresponde a los objetivos preestablecidos por la organización. El deber de actuar de forma eficaz, tiene como consecuencia también la necesidad de actuar de forma eficiente, es decir, que del principio de eficacia se derivan también el criterio de eficiencia. La cultura de Seguridad es el ambiente que se despliega de las acciones de seguridad del paciente dado en un entorno de confidencialidad y de confianza entre pacientes, profesionales, aseguradores y la comunidad. Es deber de los diferentes actores del sistema facilitar las condiciones que permitan dicho ambiente para una atención segura.

\section{Papel de la gobernanza en el Programa de Seguridad del Paciente de una Empresa social del Estado}

En esta sección se responde al objetivo de investigación formulado, desde la perspectiva de los actores que participan en el programa de seguridad del paciente en una empresa social del estado colombiano 


\subsection{Caracterización de los actores}

características de las integrantes del equipo líder de enfermería.

El siguiente cuadro muestra las

\begin{tabular}{|c|c|}
\hline \multicolumn{2}{|c|}{$\begin{array}{l}\text { Cuadro } 1 . \\
\text { Caracterización de los actores }\end{array}$} \\
\hline Tipo de actor & $\begin{array}{l}\text { Enfermeras Coordinadoras de área - EECA-; } \\
\text { Enfermeras Especialistas -EE- } \\
\text { Enfermeras Profesionales-EP. }\end{array}$ \\
\hline Edad & $\begin{array}{l}\text { Mujeres, los rangos de edad oscilan entre } 42 \text { y } 65 \text { años, con } \\
\text { una media de } 52 \text { años y mediana de } 54 \text { años. }\end{array}$ \\
\hline Estudios Posgrado & $\begin{array}{l}\text { Las EECA y EE, tienen estudios de postgrado, en su mayoría } \\
\text { maestrías del área Administrativa, una de ellas tiene doctora- } \\
\text { do. Las EP no cuentan con postgrado }\end{array}$ \\
\hline $\begin{array}{l}\text { Estudios sobre seguridad del } \\
\text { paciente }\end{array}$ & $\begin{array}{l}\text { Estudios de tipo informal sobre la Política De Seguridad Del } \\
\text { Paciente, especialmente en metas de seguridad del paciente } \\
\text { y eventos adversos. }\end{array}$ \\
\hline Promedio de años en el cargo & $\begin{array}{l}\text { Las EECA tienen un promedio de } 15 \text { años en el cargo } \\
\text { Las EE tienen un promedio de } 10 \text { años y las EP un promedio } \\
\text { de } 5 \text { años. }\end{array}$ \\
\hline Acceso al cargo & $\begin{array}{l}\text { Las EECA y EE por concurso abierto de la Comisión Nacional } \\
\text { de Servicio Civil, las EP por delegación del jefe inmediato }\end{array}$ \\
\hline
\end{tabular}

Pese a la formación de posgrado, las enfermeras con mayor grado de formación se encuentran en su etapa final de vida laboral y las más jóvenes, no alcanzan formación pos gradual; los estudios de tipo informal sobre la política de seguridad del paciente los han realizado principalmente en el tema de las metas de seguridad del paciente y eventos adversos; En conjunto, el equipo líder tiene poca formación en temas de gestión y política pública, manifestaron su el interés personal por aprender estrategias que puedan implementar para mejorar la atención segura.

Las EECA son actores formales y con autoridad sobre sus áreas de influencia en enfermeras y auxiliares de enfermería; acompañan los equipos en sus áreas, con tendencia hacia una horizontalidad en sus relaciones, pero con poca interacción con la red externa y con otros equipos. En el nombramiento de las enfermeras profesionales, EP no media un acto administrativo formal en la asignación de su función, esta informalidad le resta liderazgo e influencia en la toma de decisiones. $\mathrm{Al}$ respecto E1 afirma. "Yo veo una enfermera que me pide informes pero que puede corregirme o decirme si ella ni siquiera está nombrada".

En general el posicionamiento de todas las enfermeras entrevistadas refiere una relación de facilitador en pro de la atención segura a partir de la recolección y análisis reflexivo de acciones ligadas a su conocimiento y experiencia de la profesión, pero su 
gestión se limita por la carencia de recursos humanos, insumos y la falta de comunicación ante el nivel gerencial. Las entrevistadas, destacan la capacidad del equipo de enfermería para resolver problemas del día a día y en la atención segura.

\subsection{Principios de gobernanza por parte de los actores}

\section{Cuadro 2. Aplicación de los Principios de Gobernanza}

\begin{tabular}{|c|c|}
\hline $\begin{array}{l}\text { Participación, colabora- } \\
\text { ción y enfoque centrado } \\
\text { en el usuario }\end{array}$ & $\begin{array}{l}\text { - Involucra actores de acuerdo con las normas del PSP, con } \\
\text { visiones e intereses entre actores de la misma disciplina. La } \\
\text { aplicación presenta horizontalidad con el grupo de enfermería, } \\
\text { pero tiene limitaciones de comunicación con otras redes inter- } \\
\text { nas y a nivel externo. No tiene mucha participación a nivel cen- } \\
\text { tral, esta se remite a las visitas de los entes de control externo. } \\
\text { Reconoce las funciones y responsabilidades del conjunto de } \\
\text { actores del PSP, podrían potenciarse más la aplicación de los } \\
\text { principios con mayor articulación de los procesos del Programa. } \\
\text { A pesar de las crisis de la ESE y los cambios de personal, rea- } \\
\text { lizan acciones para que el resultado gire en función de la aten- } \\
\text { ción segura al usuario. }\end{array}$ \\
\hline $\begin{array}{l}\text { Eficacia, eficiencia y } \\
\text { cultura de la seguridad } \\
\text { del paciente }\end{array}$ & $\begin{array}{l}\text { - } \quad \text { Actúa de forma eficaz y económica en el PSP } \\
\text { - Se manifiesta un ambiente de confiabilidad y confianza entre } \\
\text { pacientes, profesionales, aseguradores y la comunidad } \\
\text { El equipo líder de enfermería, coordina el para que sea sosteni- } \\
\text { ble y se optimicen los recursos para el logro de los objetivos del } \\
\text { programa, pero para los procesos de acreditación de la ESE, } \\
\text { deben estar empoderados todos los actores. }\end{array}$ \\
\hline $\begin{array}{l}\text { Transparencia, rendición } \\
\text { de cuentas y validez }\end{array}$ & $\begin{array}{l}\text { - Realiza, analiza y socializa los resultados e indicadores del } \\
\text { PSP con trasparencia y oportunidad en la entrega. } \\
\text { - Aplica los procesos reglamentarios del PSP ofreciendo un trato } \\
\text { justo y equitativo a las partes involucradas } \\
\text { - Aplica metodología y herramientas prácticas que garanticen la } \\
\text { evidencia científica e integra la política de atención de manera } \\
\text { trasversal a todos sus componentes. }\end{array}$ \\
\hline
\end{tabular}

Fuente: Elaboración propia

El grupo investigado es un actor clave para el Programa de Seguridad del Paciente, empoderado en cuanto a la voluntad, compromiso, pero su dinámica se encuentra limitada por factores externos y dependientes del nivel directivo. El principio de participación se ha afectado por la crisis administrativa, política y financiera por los cambios de personal, es un principio que requiere de un trabajo muy profundo en cuanto a la cohesión con otros equipos médicos, trabajo social, etc.

E.4" No hay participación, yo como coordinadora de enfermería he vivido muchas experiencias en que nos tienen en cuenta por momentos, cuando los agobia los entes de control cuentan con 
nosotras, el mapa de proceso hasta la semana pasada lo lideraban los médicos directores de área y ellos son los únicos que tienen acceso a participar, en muy pocas ocasiones nosotras vamos pero no somos tenidas en cuenta, la participación es cada vez menor".

En cuanto al principio de eficacia, cultura y seguridad del paciente, según las entrevistadas; reporta mejoría de acuerdo al compromiso y aprehensión del equipo con la cultura y seguridad del paciente, pero teniendo en cuenta la interdisciplinariedad de la cultura de seguridad del paciente, E: 3 "le falta mucho por mejorar. Enfermería ha sido y es ese actor suficientemente dominante porque tiene el control sobre la atención segura, pese a carecer de suficientes recursos, ha mostrado control de los recurso necesarios y suficientes para alcanzar una cultura de seguridad del paciente en su área de servicio y en consecuencia ha logrado centrar su gobernabilidad y motivación en el control de ese capital social representado en los resultados clínicos que se tienen en el paciente, familia y entorno".

En cuanto a la rendición de informes, transparencia y validez, E6 señala. "La participación en enfermería de la ESE está dada por los informes que hay que rendir, está limitada, puede ser mayor si existiera un líder empoderado y con responsabilidad que articule lo que hacemos y cumplimos en línea con la gerencia y el entorno en que se presta la atención segura".

\subsection{Espacios, normas y procesos del programa de seguridad del paciente.}

\section{Cuadro 3. \\ Espacios, Normas y Procesos del Programa de Seguridad del paciente}

\begin{tabular}{ll}
\hline Hay suficientes espacios para el reporte de eventos adversos, ma- \\
nuales a través de los formatos, virtuales a través de la intranet y \\
plataforma de eventos, incluso correo electrónico. \\
Existen espacios para el análisis de los eventos adversos: comités \\
de análisis por áreas de atención, bimensuales o mensuales, son \\
principalmente de enfermería, tiene poca asistencia del personal de \\
otras disciplinas. \\
Espacios e interacciones \\
de enfermería en el PSP & Comités académicos donde se presentan los casos de morbimorta- \\
& lidad extrema o casos centinela \\
Las rondas de seguridad por parte de EECA, son una estrategia que \\
arroja buenos resultados. \\
Hay un comité institucional, pero tiene fechas abiertas y no cuenta \\
con un cronograma institucional de reuniones, metas y acciones a \\
seguir.
\end{tabular}




\section{Cont... Cuadro 3.}

\begin{tabular}{ll}
\hline & El programa como norma formal, El grupo de enfermería tiene \\
buena adherencia a la normatividad del Programa, pero esta adhe- \\
rencia se ha visto afectada por los cambios del personal de enfer- \\
mería, particularmente a nivel de jefes de servicio. \\
Las guías de las buenas prácticas del PSP es una estrategia peda- \\
gógica muy útil para la toma de decisiones de la ESE, tiene poco \\
nivel de consulta y le falta actualización en algunos aspectos.
\end{tabular}

Fuente. Elaboración propia.

\subsection{Espacios de interacción}

El equipo líder interactúa ante los eventos adversos pero no hay interacción con otros equipos, no se evidencia la presencia del comité institucional de eventos adversos, las reuniones del equipo $o$ de las enfermeras en los servicios no tienen impacto a nivel institucional, no se moldea la gobernanza en torno a la reducción de la inseguridad y promoción del intercambio de información. E2: "No existen espacios de interacción a nivel institucional donde se congregue a toda la comunidad a informar sobre los avances, dificultades o motivaciones que impulse la información de donde estamos y a dónde vamos".

\subsection{Normas frente al Programa de Seguridad del paciente.}

Enfermería como actor en primera línea de atención es quien incorpora normas, reglas, y otras redes de actores de acuerdo con las normas y pautas que en cada momento se establezcan para la atención centrada en el usuario, en este sentido, es importante la forma en que se operativizan las normas entre los actores y en los espacios de interacción. El programa como norma formal se implementa por parte del equipo líder como la columna vertebral de la atención segura. Las enfermeras expresan una total adherencia a la operativización del programa y consideran la guía de buenas 
prácticas clínicas como la herramienta más importante, sin embargo, la poca coordinación y comunicación con la dirección de la empresa y otros equipos profesionales frente al proceso generan una informalidad en la normativa para su implementación.

\subsection{Los procesos}

El proceso más importante para la toma de decisiones respecto a la atención segura, es el reporte de los eventos adversos y la implementación de los planes de mejoramiento. El principal proceso del programa que leva a acabo el grupo líder, se refiere al reporte de los eventos adversos, E.9 explica, "Los reportamos trimestral, semestral y al año, nos reunimos y revisamos siempre con el medico de seguridad del paciente del servicio, invitamos una enfermera y yo, algunas veces la médica jefe del servicio, además los socializamos y publicamos el informe en cada sala, y por sala hacemos los indicadores cuál fue el evento que más se presentó, los indicadores se envían a nivel central, pero nunca hay retroalimentación desde la dirección". E3 "la poca cultura de hacer una actividad que es de todos, recae solo en enfermería, que si bien ha logrado resultados como una comunicación más abierta, no resulta ser suficiente para la continuidad del proceso de manera continua, coherente y con inversión y responsabilidad en todos los niveles y por todos los actores".

En términos generales, el marco analítico para la gobernanza en Salud, permite un proceso pragmático de interacción entre los actores involucrados, en este caso en un programa de Seguridad del Paciente, dependiente de una política pública. Una perspectiva con énfasis en los actores es fundamental para garantizar una atención segura en una ESE. Hufty (2006).

Las actuaciones del grupo de enfermería, como líder del programa, influyen en la toma de decisiones, promoviendo los espació de interacción con los actores, en la adherencia a las normas formales e informales y en el proceso relacionados con los eventos adversos, su reporte, mejoramiento, comunicación de los eventos adversos, convirtiéndolo en un actor estratégico (Prats, 2002) para la atención segura en salud.

El equipo líder, cuenta en su mayor parte con formación de postgrado, pero su formación en la política pública responde a capacitaciones de tipo informal. Esto es un hecho relevante, dada la necesidad de acreditación en alta calidad de las empresas de salud, planteada por el Ministerio de salud desde el 2005. (Minsalud, 2016). Los actores manifestaron liderazgo, coordinación, capacidad gerencial y administrativa en el programa, pero no gobernanza. Por otra parte, no hay espacios de interacción con otros equipos de la institución y las directivas, lo que implica que la transversalidad del programa, se vea afectada. (Minsalud, 2008)

Los principios de gobernanza son importantes para la toma de decisiones e involucran todos los actores, (Cerillo I Martinez, 2005), se destaca su horizontalidad en el nivel de inmediata influencia, pero la informalidad en la función del cargo y la falta de coordinación, impactan en la cultura de seguridad del paciente de manera institucional. En países como Colombia, las relaciones entre los actores, las crisis financieras, los procesos para la tomas de decisiones, entre otros factores, 
manifiestan un problema de pertinencia entre un modelo centrado en el mercado y un contexto que trata de garantizar la salud como servicio público. (Rojas, 2017, 56).

Los espacios de encuentro, enfatizan los reportes e indicadores de los eventos adversos y no cuentan con una programación planificada que retroalimente las propuestas de mejoramiento. involucre todos los actores y las directivas, para hacer amplia la discusión de problemas, procesos, actores en relación al programa de seguridad del paciente, no corresponde, al cruce de universos de significación o visiones del mundo que permita una puerta de entrada a los procesos de gobernanza. (Hufty, 2010). Las normas informales si bien permiten la operatividad del programa, requieren formalizarse para mejorar las prácticas, identificar sus características, relaciones y el efecto sobre el proceso de cambio como acción urgente para la acreditación en atención segura (Minsalud, 2016).

Para Hufty (2010), las normas son las expectativas colectivas que hacen referencia al comportamiento aceptado en una sociedad, enfermería se alinea en este marco teórico en el proceso de acreditación dado que posee: primero, los elementos de valores y creencias en el sentido de lo bueno o incorrecto; segundo, sugiere lo que uno tiene que hacer o no y tercero supone sanciones positivas o negativas facilitando las relaciones y reproduciendo en la sociedad la norma de seguridad del paciente. La experiencia y conocimientos del equipo, son fortalezas para la adherencia del programa, porque ya tiene cultura y evolución muy importante, una atención segura para cuidar y preservar la vida que hace parte de la vocación disciplinar,(ley 266,1996ría) y en el marco del plan estratégico que define el comité internacional de enfermería (CIE, 2014).

Una fortaleza de este estudio, Desde la perspectiva de la gobernanza, el equipo líder tiene una gobernanza débil, con una red de actores pobre, pero las enfermeras de la ESE pese a sus debilidades, pueden ganar más autonomía basada en el conocimiento y asumiendo una actitud de liderazgo; en su área interna de trabajo que crea cultura de seguridad en el día a día, seguimiento, y estudio de los eventos adversos. Como limitación de este estudio, si bien se consultó al equipo líder del programa, por limitaciones de tiempo no se entrevistaron actores de interés como auxiliares y enfermeras.

Si bien se identifica la falta de gobernanza en el equipo líder del programa, la falta de una acción conjunta y planificada institucionalmente frente al programa no es menor, sin embargo, dentro de este marco ha de considerarse que los valores disciplinares, la cultura organizacional y visión de la E.S.E crean un objetivo estratégico real y contundente para la acreditación nacional.

\section{Conclusiones}

La política de seguridad del paciente contiene unos lineamentos mínimos como estrategias para cumplirse en las instituciones mediante la implementación del programa de seguridad del paciente que garantice las buenas prácticas y por consiguiente la atención segura. Por esta razón la aplicación de principios de gobernanza como factor transversal en la coordinación entre el Estado, las instituciones y sus actores es claves 
para la generación de modelos de gobernanza que mejoren la capacidad estratégica del Estado y la acreditación en atención segura de las instituciones a nivel nacional e internacional.

Enfermería, como actor principal del cuidado propende objetivos de cuidado integral (persona-familiacomunidad), es prioritario involucrar su actuar bajo principios de gobernanza articulados a los definidos por la ética de enfermería en el código deontológico de enfermería, la comunidad internacional de enfermería y las políticas públicas como una estrategia esencial para generar modelos de gobernanza.

El grupo de Enfermería en la ESE tiene una gran oportunidad para ejecutar un modelo de gobernanza a partir de la visión, conocimiento y estrategia de atención segura, que empodere a todos los actores en principios de gobernanza e impacte en el cumplimiento de la norma que supone establecer oportunidades significativas de agentes claves del sector de usuarios, científico, económico, social, entre otros, para que en red se direccione la política pública y el diseño de procesos de atención segura necesario para la acreditación de la empresa y el desarrollo institucional, disciplinar y personal.

De igual manera, en cuanto a los cargos y los actos administrativos de las personas que tienen esta función en seguridad del paciente, hay algunas personas que lo hacen sin que medie un acto administrativo, lo que le resta fortaleza a la posibilidad de una gobernanza con liderazgo, participativa, una gobernanza con excelente toma de decisiones. En este sentido se puede también concluir que el recurso humano que tiene a cargo el programa de seguridad del paciente tiene debilidades relacionadas con los cambios y las crisis que ha tenido la ESE en la que se realiza la investigación, esto ha afectado principalmente al talento humano de enfermería quien tiene a cargo las acciones de la política de seguridad del paciente, a la vez que presenta fortalezas en cuanto a su larga experiencia y formación pos gradual, pero tiene debilidades en la formación en los campos específicos de la Calidad y debilidad en cuanto a alguna funciones que deben ser formalizadas mediante acto administrativo, no pueden ser asignadas de manera informal o por aclamación,

La red de actores no la hay como elemento de la cultura organizacional, este elemento es clave para la acreditación institucional, pero se cruza con el compromiso y colaboración como elemento de la cultura de seguridad del paciente, enfermería interactúa ante los eventos adversos, pero no hay interacción con otros equipos, no se evidencia la presencia del comité institucional de eventos adversos, restando impacto a nivel institucional. La gobernanza del quipo líder del programa es débil, al estar inmerso en una institución que sufre las tensiones y limitaciones del sector público en salud del país.

\section{Referencias Bibliográficas}

Aguilar, Luis F. (2003), Gobernanza y gestión pública. Fondo de Cultura Económica, México D.F.

Aguilar, Luis F. (2007), El aporte de la política pública y la nueva gestión pública a la gobernanza. Congreso Internacional del CLAD sobre la reforma del Estado y de la Administración Pública, Santo Domingo, Rep. Dominicana, 30 de octubre - 02 de noviembre. 
Gobernanza, enfermería, seguridad del paciente en una empresa social del estado colombiano

Mera García, Manuela; Thorpt Hurtado, Carmen del Pilar; González Osorio, María Fernanda

Banco Mundial. BM, (2017), Informe sobre el desarrollo mundial 2017: La gobernanza y las leyes, cuaderniIlo del "Panorama general", Banco Mundial, Washington DC. Licencia: Creative Commons de Reconocimiento CC BY 3.0 IGO.

Cardona , A., Nieto, E., \& Mejia, L. M. (2010). Un enfoque de gobernanza aplicado al anàlisis de una investigacion sobre trabajadores en Medellin, Colombia. Salud Pùblica, Pp 123 137.

Cerillo i Martinez, A. (2005). La Gobernanza hoy: 10 textos de referencia. Madrid: Instituto Nacional de Administraciòn Pùblica (Estudios Goberna). Pp 11-36

Comité Internacional de Enfermería, CIE, C. I. (2014), Plan Estrategico del CIE: 2014- 2018. Ginebra, Suiza

Draga Ramírez, L., García Vargas, S., \& Trujillo Sarmiento, A. G. (2016). Percepción de necesidades de humanización en personal asistencial de salud vs personal administrativo en una clínica de Cali. Pontificia Universidad Javeriana, Cali.

Díaz-Castro, L., Arredondo, A., Pelcastre Villafuerte, B. E., \& Hufty, M. (2017), Indicadores de gobernanza en políticas y programas de salud mental en México: una perspectiva de actores clave. Gaceta Sanitaria, 31 (4), Pp 305-312. https://doi.org/10.1016/j.gaceta.2016.08.001

Gomez Ramirez, O., Arenas Gutièrrez, W., Gonzalez Vega, L., Garzon Salamanca, J., Mateus Galeano, E., \& Soto Gomez, A. ( 2011). Cultura de Seguridad del Paciente por Personal de Enfermerìa Bogotà Colombia. Ciencia y Enfermerìa Vol. 17, Vol 3, Pp 97 - 111

Guedes dos Santos, J., \& Lorenzini Erdmann, A. (2015). Governance of pro- fessional nursing practice in a hospital setting: a mixed methods study. Rev. Latino-Am. Enfermagem [online]. 2015, vol.23, n.6, Pp.10241032. ISSN 0104-1169. http://dx.doi. org/10.1590/0104-1169.0482.2645

Hufty, Mark. (2006), Marco conceptual y analítico de la gobernanza. Documento Congreso Mundial de Salud Pública. Recuperado de http://www.idrc.ca/uploads/ user-S/11556776031Paper_Hufty_ Fr.doc.

Hufty, Mark. (2010). Gobernanza en salud pública: hacia un marco analitico. Revista de Salud Publica, Volumen 12, (1),Pp 39-61.

Kooimann, Jan. (2005), Gobernar en Gobernanza. En A. C. Martinez, 10 Textos de Gobernanza ( 57-82). Madrid: Instituto Nacional de la Administraciòn Pùblica.

Congreso de Colombia (2000), Ley 550 de 1990. Diario Oficial No. 43.940, de 19 de marzo de 2000.Bogotá , Colombia.

Congreso de Colombia (1996), Ley 266 de 1996. Ley de Enfermería. Diario Oficial No. 42.710 del 5 de febrero de 1996.Bogotá, Colombia

Ministerio de Salud y Proteccion Social(2016), OCDE. Guia Metodologica:Sistema de Evaluación del Desempeño del Sistema de Salud de Colombia bajo Criterios de la OCDE. Bogota DC: Min. Salud.

Minsalud, M. d. (2008), Lineamientos de la Politica de Seguridad del Paciente. Bogota: Ministerio de Salud y de la Proteccion Social.

Minsalud, M. d. (2011), Guìa Tècnica de Buenas Practicas en Seguridad del Paciente. Bogotà: Minsalud.

Minsalud, M. d. (2011), Paquete: Promociòn de la Cultura de Seguridad 
del Paciente. Bogotà: Minsalud.

Minsalud, (2012), Resolución 112 del 2002. Lineamientos para la implementación de la Política de Seguridad del Paciente. https://www. minsalud.gov.co/.../Resoluciòn $\% 20$ $0112 \% 20$ de $\% 202012 \% 20$ - \% 20 Docu...

OECD (2017), Estudio de la OCDE sobre integridad en Colombia: Invirtiendo en integridad pública para afianzar la paz y el desarrollo, Estudios de la OCDE sobre Gobernanza Pública, OECD Publishing, Paris, https://doi.org/10.1787/9789264278646-es.

Organización Mundia de Salud, OMS. (2009). Estudio Ibeas.Hacia una Atención Hospitalaria más Segura. Prevalencia De Efectos Adversos En Hospitales De Latinoamerica. OMS. https://www.who.int/patientsafety/research/ibeas_report_es.pdf

Prats, Joan. (2002): La Gobernabilidad local en la Sociedad de la información y el conocimiento, UOC, Barcelona.

Prats, Joan. (2005). Modos de Gobernacion de la Sociedades Globales. En A. C. Martinez, 10Textos de Gobernanza. Pp 145, 172. Madrid: Instituto Nacional de Administraciòn Pùblica.

Rojas, Paula, (2017), Gobernanza en salud.Estudio de caso: Un análisis del sistema de protección. Tesis de grado para optar el título de politóloga, Universidad Javeriana, Bogotá.

Subirast, Joan. (2010), Si la respuesta es gobernanza, ¿cuál es la pregunta? factores de cambio en la política y en las políticas. Ekonomiaz: Revista vasca de economía, ISSN 02133865, No.74, Pp16-35_

WHO (1998), Good governance for health. WHO/CHS/HSS98.2. World Health Organization. Geneva.

- Esta obra está bajo una licencia de Creative Commons Reconocimiento-NoComercialCompartirlgual 3.0 Unported. http://creativecommons.org/licenses/by-nc-sa/3.0/deed.es_ES 